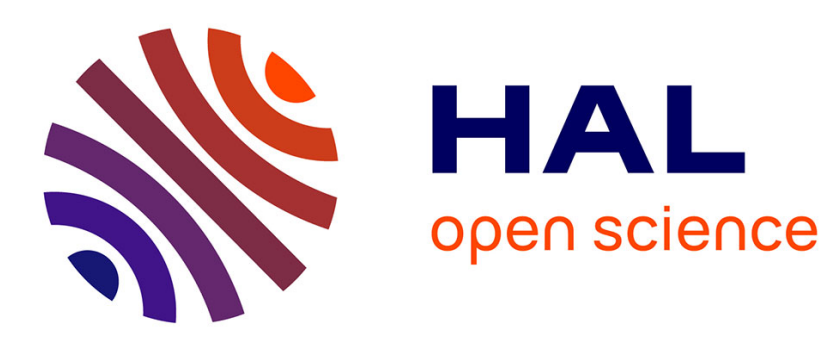

\title{
Calculation and analysis of assembly clearance based on nonideal surface discrete data
}

\author{
Yue Li, Lihong Qiao, Uzair Khaleeq Uz Zaman, Ali Siadat
}

\section{To cite this version:}

Yue Li, Lihong Qiao, Uzair Khaleeq Uz Zaman, Ali Siadat. Calculation and analysis of assembly clearance based on nonideal surface discrete data. International Journal of Modeling, Simulation, and Scientific Computing, 2017, 08 (02), pp.1750050. 10.1142/S1793962317500507 . hal-02434830

\section{HAL Id: hal-02434830 \\ https://hal.science/hal-02434830}

Submitted on 10 Jan 2020

HAL is a multi-disciplinary open access archive for the deposit and dissemination of scientific research documents, whether they are published or not. The documents may come from teaching and research institutions in France or abroad, or from public or private research centers.
L'archive ouverte pluridisciplinaire HAL, est destinée au dépôt et à la diffusion de documents scientifiques de niveau recherche, publiés ou non, émanant des établissements d'enseignement et de recherche français ou étrangers, des laboratoires publics ou privés. 


\title{
[AsiaSim] Calculation and analysis of assembly clearance
}

\section{based on non-ideal surface discrete data}

\author{
Yue $\mathrm{Li}^{1}$, Lihong Qiao ${ }^{1}$, Uzair Khaleeq uz Zaman ${ }^{2}$, Ali Siadat ${ }^{2}$ \\ ( ${ }^{1}$ School of Mechanical Engineering and Automation, Beihang University; Engineering \\ Research Center of Complex Product Advanced Manufacturing System, Ministry of Education, \\ China. ${ }^{2}$ Laboratoire de Conception Fabrication Commande (LCFC), Ecole Nationale Supérieure \\ d'Arts et Métiers (ENSAM), Metz, France.)
}

\begin{abstract}
Calculation of assembly clearance is the basis of analysis on assembly problems, and the real assembly surface is the non-ideal surface. In this paper, the concept of assembly clearance on non-ideal surface is defined and the calculation algorithm of assembly clearance is designed. Based on the calculation, the concept of clearance surface is put forward to express the distribution of assembly clearance on non-ideal surface as it can assist the analysis of assembly problems. Finally, the effectiveness of the algorithm is verified by the calculation of the assembly clearance between the surfaces of piston and cylinder.
\end{abstract}

Keywords: assembly problem, non-ideal surface, clearance surface

\section{Introduction}

In assembly processes, assembly problems arise due to geometric deviation of the surface of mechanical parts. Such problems are generally categorized in to two aspects; product performance and assembly process. The former problem is caused by the deviation of assembly surfaces such as the sealing problem and the friction problem, while the latter assembly problem include the assembly method, path and other issues such as interference problem and assembly path planning problem.

In the simulation study of assembly problem due to geometric deviation, traditional $\mathrm{CAD} / \mathrm{CAM} / \mathrm{CAE}$ simulation tools usually describe surface of parts as ideal model, where the geometric deviation is over simplified, and the shape of surface can't be accurately described. 
In order to describe the changes of the surface accurately, this paper uses non-ideal surface model to express the surface. Non-ideal surface model makes up the deficiency of the ideal surface model which contains the geometric deviation from manufacturing processes [1]. The non-ideal surface model not only describes the real changes of the surface, known as the "physical interface model between the workpiece and the surrounding environment" but also accurately describes the shape and geometric deviation of assembly surface $[2,3]$.

At present, the research on non-ideal surface is still in the exploratory stage. French laboratory gives the study from geometric specification and puts forward a geometric representation scheme for non-ideal surface. Zhang et al. proposed to use quadric surface and statistical methods to obtain the random geometric deviation of the product [4]. Moreover, Schleich et al. worked on an assembly motion simulation framework which was based on the application of non-ideal surface [5]. Researchers of Beihang University have conducted a lot of researches on non-ideal surface especially in the generative process of non-ideal surface sample data. They have also done the research on the non-ideal surface deviation representation and the application of non-ideal surface $[6,7,8]$. Song et al. present a modeling framework for product lifecycle information which can be used in product data management [9]. Bo hu li et al. present a modeling language for Complex system modeling, simulation and optimization [10].

'Assembly clearance' is referred to as the clearance between the two assembly surfaces in which the active surface is called "query surface" and the passive surface is called "datum surface". As assembly clearance is the basis of the analysis of assembly problems, it can reflect the relationship of the assembly surface. Over the years, many researchers have worked on the subject of assembly clearance. Researchers in China Academy of Engineering Physics have conducted research on assembly clearance using image measurement technology [11]. Also, the measurement system of product assembly system based on image measurement technology has been studied in Sichuan University which can realize the non-contact measurement technology of assembly clearance [12]. Furthermore, researchers have also studied the influence of the assembly clearance between the piston and the cylinder on the lubrication performance using the method of numerical analysis to determine the best clearance [13].

Keeping in view the above researches, it is imperative to understand that assembly clearance based on non-ideal surface is important to solve the assembly problem caused by the geometric deviation. The key point of the study lies in the organization of nonideal surface discrete data and the calculation of assembly clearance. 


\section{Discrete data modeling of non-ideal surface}

Discrete data modeling of non-ideal surface is a prerequisite for the simulation of non-ideal surface assembly problem. Non-ideal surface discrete data is composed of many vertexes data divided by surface meshes. These data include spatial coordinates $(x, y, z)$ and grid position coordinates $(i, j)$ of these vertices. Point cloud model and triangulated-surface model are used in this paper to model non-ideal surface based on non-ideal surface discrete data. Point cloud model is the organization of the sampling points on the non-ideal surface which orders point data in accordance with the grid position coordinates $(i, j)$ to get each point element and calculate with it. Point cloud model is used to model query surface.

When the clearance between non-ideal surfaces is calculated, the clearance between the point on the query surface and datum surface may occur in the gaps between the discrete points of datum surface. Here, the triangle facet model can process discrete point in a continuous manner. Also, the triangle surface model applied in this paper first combines and processes point data of non-ideal surface sample and then forms more high-level geometric elements to achieve the continuity of the surface. Elements in triangle facet model includes point, edge, facet, vector and surface. Overall design is as shown in Figure 1.

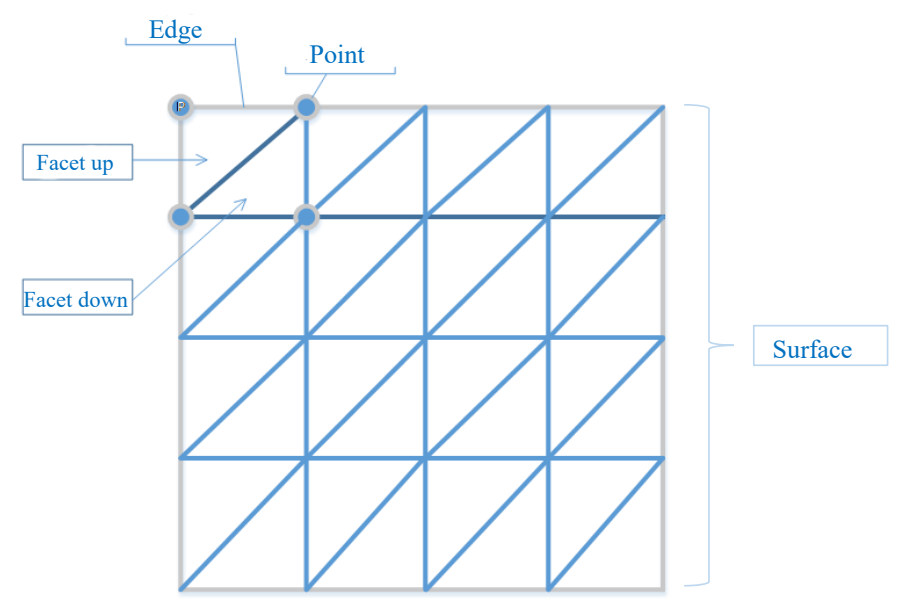

Fig.1. Facet model

Point of the model records 3D coordinate information of sample points and the grid coordinates $(i, j)$. Each grid is divided into two triangle facets as the same way. These two triangle facets are mated. Moreover, one triangle facet records three points and three edges of it. Each edge of the model contains two points of facet. Vector in this 
model is an assistant to represent the direction of edge and normal of facet. Vector contains three direction values of it. Furthermore, surface of the model contains all the points and facets on non-ideal surface. It is the highest level in the model.

Point cloud model and triangle facet model are used to model the query surface and the datum surface, respectively. The calculation based on these models will be proposed in the following section.

\section{Calculation and analysis of assembly clearance}

\subsection{Definition and calculation of non-ideal surface clearance}

For the analysis of assembly problem, it's important to calculate the assembly clearance. The concept definition of assembly clearance is the first problem to solve. Assembly clearance refers to the clearance between two assembly surfaces, and because of the non-ideal surfaces, assembly clearances are different at each places on assembly surfaces. Furthermore, assembly clearance concepts are different for various assembly problems. For sealing and friction problems, etc., the clearance refers to the shortest distance between the point on query surface and the datum surface, as shown in Figure 2. For assembly path planning and other issues, the clearance refers to the distance of the point on query surface to the datum surface in the assembly direction, as shown in Figure 3.

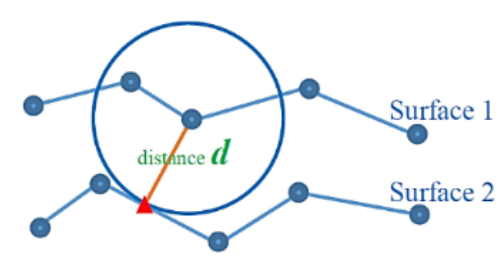

Fig.2. The shortest distance

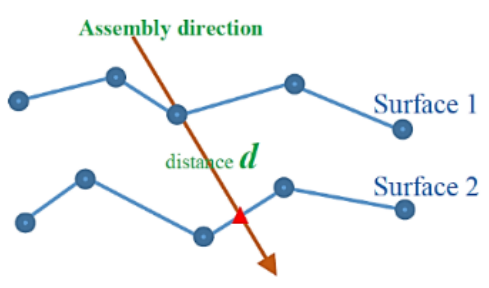

Fig.3. Clearance in assembly direction

Two clearance calculation algorithms are designed in this paper for different concepts of assembly clearance; one, for the calculation of clearance in the sense of shortest distance and the other for the calculation of clearance in assembly clearance. Both are explained as follows:

\section{1) Algorithm for assembly clearance of shortest distance}

The design ideas of algorithm stem from the calculation of relationship between 
geometrical elements to the calculation of clearance. The process is described in the paragraph to follow.

First, the shortest distance between a point and a triangle facet is calculated. It is required to judge here whether the projection point is inside the facet or not. Then the query point is set as $P_{0}(x, y, z)$ and three points of facet are fixed as $A(x 1, y 1, z 1)$, $B(x 2, y 2, z 2), C(x 3, y 3, z 3)$. Then the normal vector of facet is obtained as $V(a$, $b, c)$ and the coordinate of projection is set as $P(X, Y, Z)$.

$$
\begin{aligned}
& d=a \bullet x 1+b \bullet y 1+c \bullet z 1 \\
& k=(d-a \bullet x-b \bullet y-c \bullet z) /\left(a^{2}+b^{2}+c^{2}\right) \\
& \left\{\begin{array}{l}
X=x+k \bullet a \\
Y=y+k \bullet b \\
Z=z+k \bullet c
\end{array}\right.
\end{aligned}
$$

The method of barycenter is used to determine whether the projection point is within the triangle facet, the judgement condition is as follows.

$$
\begin{aligned}
& P=A+\mu(C-A)+v(B-A) \\
& \left\{\begin{array}{l}
\mu \geq 0 \\
v \geq 0 \\
\mu+v \leq 1
\end{array}\right.
\end{aligned}
$$

If the projection point within the facet, then shortest distance will be the projection distance $D$.

$$
\begin{aligned}
& d=a \bullet x 1+b \bullet y 1+c \bullet z 1 \\
& D=(a \bullet x+b \bullet y+c \bullet z-d) / \sqrt{\left(a^{2}+b^{2}+c^{2}\right)}
\end{aligned}
$$

If the projection point is outside the facet, the shortest distance will occur between the point and the edge of facet. Then it needs to calculate the distance between the point $P$ and an edge. Therefore, set the edge as $(A, B)$ and the distance between three points $P, A, B$ are $P A, P B$, and $A B$, respectively. The shortest distance is $d_{l}$.

If $P A^{2}>P B^{2}+A B^{2}$, then $d_{1}=P B$, if $P B^{2}>P A^{2}+A B^{2}$, then $d_{1}=P A$, otherwise, the result is as follows:

$$
\left\{\begin{array}{l}
L=(P A+P B+P C) / 2 \\
d_{1}=2 \times \sqrt{L(L-P A)(L-P B)(L-A B)} / C
\end{array}\right.
$$

Furthermore, the three distances of three edges to the point are calculated as the 
shortest distance $D$.

$$
D=\min \left(d_{1}, d_{2}, d_{3}\right)
$$

After getting the shortest distance $D$ between the point and the facet, all facets on the datum surface are traversed to calculate the distances $\left\{D_{1}, D_{2}, \ldots D_{n}\right\}$. Then the clearance between the point on query surface and the datum surface is as follow.

$$
\text { clearance }=\min \left\{D_{1}, D_{2}, \ldots D_{n}\right\}
$$

Where $n$ is the number of facets and the clearance value is the minimum value of the distance between a query point and all facets on datum surface.

The calculation result shows that under a certain datum surface, each point on the query surface is corresponding to a clearance value. It is a kind of mapping relationship. The relationship can be expressed as follow.

$$
\text { clearance }=f(i, j)
$$

2) Algorithm for assembly clearance in assembly direction

The algorithm for assembly clearance in assembly direction is different from the previous algorithm. It needs to find the facet which was through by the point $P_{0}(x, y, z)$ in the assembly direction $V(a 1, b 1, c 1)$ For example, to determine whether the point is through facet $\triangle A B C$ in the direction. The points are $A(x 1, y 1, z 1)$, $B(x 2, y 2, z 2), C(x 3, y 3, z 3)$, the normal vector of facet is obtained as $V(a 0, b 0, c 0)$. First, calculate the projection point in assembly direction as $P^{\prime}(X, Y, Z)$.

$$
\begin{aligned}
& k=a 0 \bullet a 1+b 0 \bullet b 1+c 0 \bullet c 1 \\
& t=(x 1-x) \bullet a 0+(y 1-y) \bullet b 0+(z 1-z) \bullet c 0 \\
& \left\{\begin{array}{l}
X=x+a 1 \bullet t \\
Y=y+a 2 \bullet t \\
Z=z+a 3 \bullet t
\end{array}\right.
\end{aligned}
$$

Then using the method above to judge whether the projection point is within the facet. All the facets are traversed to find the facet which projection point within. Then the clearance in assembly direction is as follow.

$$
\text { clearance }=\left|P_{0} P^{\prime}\right|=\sqrt{(x-X)^{2}+(y-Y)^{2}+(z-Z)^{2}}
$$

In the formula, the clearance value is the distance between the query point and the projection point. The calculation result shows that, under a certain datum surface, each point on the query surface is corresponding to a clearance value. It is a kind of mapping 
relationship. The relationship can be expressed as follow.

$$
\text { clearance }=f(i, j)
$$

\subsection{Clearance surface analysis method}

Each point on the query surface corresponds to a clearance value. Meanwhile, each point corresponds to its grid position coordinate $(i, j)$ on the surface. This correspondence is a mapping relationship, expressed as clearance $=f(i, j)$. This formula represents a functional relationship between the clearance and the grid coordinates of the point. And because the grid coordinates of the points express the distribution on the non-ideal surface, this formula represents the distribution of clearances on the non-ideal surface. This formula is a function of three variables, which can be understood as a kind of surface relationship, so the concept of clearance surface is used to represent the surface relationship in this paper.

The construction process of the clearance surface is shown in Figure 4.

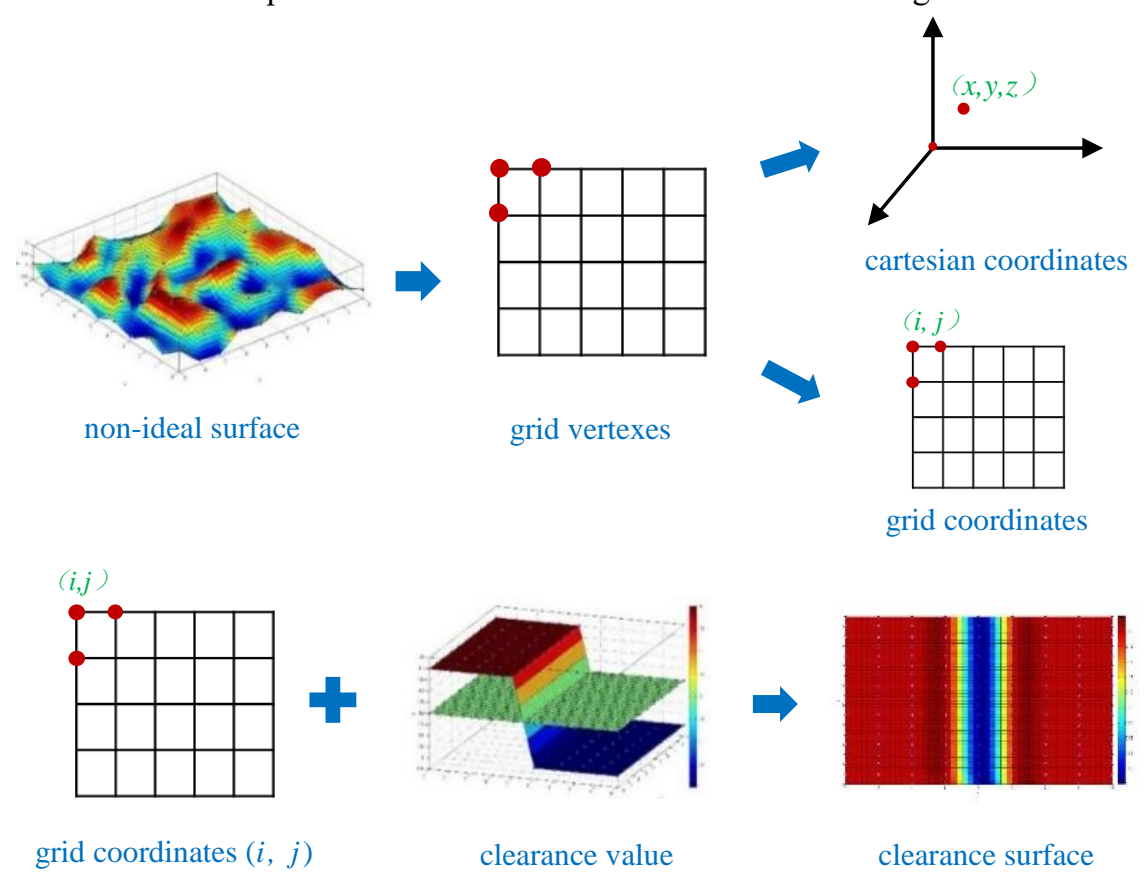

Fig.4. Construction process of clearance surface

The clearance surface is related to the clearance value to the position coordinates of the points on the non-ideal surface based on the calculation of clearance value. The distribution law of the clearance value on the non-ideal surface is reflected in this way. 
The clearance surface analysis method is proposed in this paper. Using clearance surface to reflect the distribution law of the clearance, gaining the information of assembly surface position for assembly problem analysis.

In order to show the distribution law of clearance more intuitively, the data visualization of the calculated clearance surface is carried out in this paper. The clearance surface data is processed in MATLAB. With clearance value as the $\mathrm{Z}$ axis, the grid coordinates of the point are the $\mathrm{X}$ axis and the $\mathrm{Y}$ axis, and the space surface image is drawn. The value of the clearance is differed from the color difference, finally a graphical clearance surface with colorful layer is formed, which can assist the analysis of assembly problem by using the distribution of color to reflect distribution of clearance.

\section{Application of algorithm with calculating the non-ideal surface assembly clearance}

The assembly clearance between the surfaces of piston and cylinder were calculated and analyzed by means of the assembly clearance algorithm and the clearance surface analysis method in this paper. Assembly clearance between piston and cylinder refers to the clearance between the piston skirt and cylinder. Moreover, the sample point data on non-ideal surfaces of piston and cylinder were utilized at first to calculate assembly clearances and afterwards, the assembling clearances were generated and displayed visually, which made it possible to analyze the assembly problem with graphical clearance surface.

The picture of calculated clearance surfaces is shown in Figure 5.

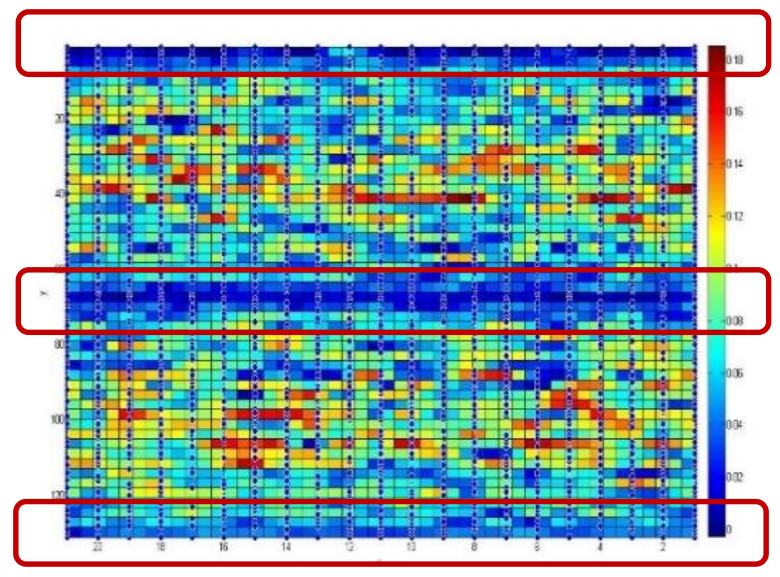


Fig.5. Clearance surface between piston and cylinder

The clearances are not distributed equally by analyzing the clearance surface picture. There are red areas in the figure which refer to large clearances, where the leak may happen. In contrast, too small clearances are marked with blue strips (the selected areas in the middle and on two sides in this figure). There would be the fiction between piston and cylinder.

It can be seen from the analysis that the relationship between assembly surfaces is described through the clearance surface, then the assembly problems can be analyzed. Next stage, calculation algorithm need to be more accuracy and higher speed. It is possible to make a more accurate analysis with the clearance surface, for example to determine the specific position of interference and interference area, etc.

\section{Conclusion}

The point cloud model and triangle facet model are used to model the non-ideal surface data, which enabled the accurate and valid computation of assembly clearance. For the clearance of shortest distance and assembly direction respectively, two algorithms were designed in order to calculate the assembly clearance between nonideal assembling surfaces. The concept of clearance surface was proposed to present the functional relationship of clearance and the grid coordinates of point. The distribution of assembling clearance on non-ideal surfaces was also presented by clearance surface that could help with the research of assembly problem. In conclusion, this paper provided a method for calculating and analyzing assembly clearance, basing on the non-ideal surface data, which provides new ideas for assembly problem research in the case of non-ideal surface.

Acknowledgements. This work is supported by National Natural Science Foundation of China (Grant 51575031) and the key program of the Engineering Research Center of Complex Product Advanced Manufacturing System, Ministry of Education.

\section{References}

1. Zhu Zuowei. Research on assembly tolerance analysis based on skin model shapes. Beijing, Beihang University.1-84. 2016

2. Anwer N, Ballu A, Mathieu L. The skin model, a comprehensive geometric model for 
engineering design. CIRP Annals-Manufacturing Technology. 62(1), 143-146. 2013

3. Schleich B, Anwer N, Mathieu L, et al. skin model shapes: a new paradigm shift for geometric variations modelling in mechanical engineering. Computer-Aided Design. (50), 1-15. 2014

4. Zhang Min, Anwer N, Mathieu L, et al. A discrete geometry framework for geometrical product specifications. Proceedings of the 21st CIRP design conference. 36-41. 2011

5. Schleich B, Anwer N, Mathieu L, et al. Contact and mobility simulation for mechanical assemblies based on skin model shapes. Journal of Computing and Information Science in Engineering. 1-7. 2014

6. Qiao Lihong, Wu Jianshun, et al. Approach to the deviation representation of non-ideal cylindrical surfaces based on the curvilinear coordinate system. Procedia CIRP. (43) 1722. 2016

7. Orzuri Rique Garaizar, Qiao Lihong, et al. Integration of thermal effects into tolerancing using skin model shapes. Procedia CIRP. (43) 196-201. 2016

8. Cai Na, Qiao Lihong. Rigid-compliant hybrid variation modeling of sheet metal assembly with 3D generic free surface. Journal of Manufacturing Systems. (41) 45-64. 2016

9. Song Xiao, et al. Modeling framework for product lifecycle information. Simulation Modelling Practice and Theory. (8) 1080-1091. 2010

10. Bo Hu Li, Xiao Song*, Lin Zhang et al, CoSMSOL: Complex system modeling, simulation and optimization language, International Journal of Modeling, Simulation, and Scientific Computing, 1741002 (2017) [21 pages]

11. Jiang Jiadong, Yuan Daocheng, Pu Jie. Research on on-line-measuring clearance of assembly parts based on image measurement technology. Journal of Transducer Technology. (12) 26-28. 2005

12. Fu Degui. Reserch on the online measurement system of product gesture and assembly interval. Chengdu, Sichuan University.1-50. 2013

13. Yang Junwei, Yu Xudong, et al. The effects of radial clearance between piston and cylinder bore on piston lubrication. Mechanical Science and Technology. (5) 694-696. 2002 